\title{
Immunosuppressive macrophages induced by IDO1 promote the growth of endometrial stromal cells in endometriosis
}

\author{
JIE MEI $^{1}$, KAI-KAI CHANG ${ }^{2}$ and HAI-XIANG SUN ${ }^{1}$ \\ ${ }^{1}$ Reproductive Medicine Center, Department of Obstetrics and Gynecology, Nanjing Drum Tower Hospital, \\ The Affiliated Hospital of Nanjing University Medical School, Nanjing, Jiangsu 210008; ${ }^{2}$ Hospital and Institute \\ of Obstetrics and Gynecology, Shanghai Medical School, Fudan University, Shanghai 200011, P.R. China
}

Received December 19, 2015; Accepted December 22, 2016

DOI: $10.3892 / \mathrm{mmr} .2017 .6242$

\begin{abstract}
It was previously demonstrated that anomalous expression of indoleamine 2,3-dioxygenase-1 (IDO1) in endometrial stromal cells (ESCs) stimulated an inflammatory response that subsequently initiated the activation of immunosuppressive macrophages in endometriosis. The aim of the present study was to clarify the effect of IDO1-induced macrophages on the growth of ESCs in endometriosis. Normal ESCs, ectopic ESCs and normal ESCs treated with plasmid pEGFP-N1-IDO1 or SD11-IDO1 short hairpin RNA were co-cultured with peripheral blood-derived monocyte (PBMC)-driven macrophages directly for $48 \mathrm{~h}$. Compared with normal ESCs, the PBMC-driven macrophages that were co-cultured with ectopic ESCs displayed a lower phagocytic ability. pEGFP-N1-IDO1 transfection of normal ESCs also decreased the phagocytic ability of co-cultured macrophages. Additionally, pEGFP-N1-IDO1-transfected ESC-induced macrophages significantly increased the viability and proliferation of ESCs, while ESC apoptosis was decreased, compared with control ESCs. In conclusion, IDO1 educated-macrophages may facilitate the survival of retrograde endometrial tissues, and be involved in the pathogenesis of endometriosis.
\end{abstract}

\section{Introduction}

Endometriosis, which is defined by the presence of endometrial tissue outside of the uterus, occurs in $\sim 10 \%$ women, and is associated with persistent pelvic pain and infertility. Despite extensive research, the etiology of endometriosis remains elusive. Inflammation of the surrounding pelvic

Correspondence to: Dr Hai-Xiang Sun, Reproductive Medicine Center, Department of Obstetrics and Gynecology, Nanjing Drum Tower Hospital, The Affiliated Hospital of Nanjing University Medical School, 321 Zhong Shan Road, Nanjing, Jiangsu 210008, P.R. China

E-mail: stevensunz@163.com

Key words: endometriosis, indoleamine 2,3-dioxygenase, endometrial stromal cell, macrophage, cell proliferation microenvironment is thought to have an important role in the initiation and progression of endometriosis, in addition to ovarian steroid hormones (1). The ectopic growth of 'lesions', consisting of endometrial cells outside the uterine cavity, stimulate an inflammatory response, which initiates the activation of macrophages and leads to increased concentrations of cytokines and growth factors in the peritoneal fluid $(2,3)$.

Indoleamine 2,3-dioxygenase (IDO1) is an intracellular heme enzyme that catalyzes the initial and rate-limiting step in the metabolism of the essential amino acid tryptophan in the kynurenine pathway. In the last decade, numerous studies have demonstrated that IDO1 produces a marked tolerance effect in fetal rejection, organ transplantation, autoimmune disorders and cancer (4-6). We previously demonstrated that elevated IDO1 expression in eutopic and ectopic endometrial stromal cells (ESCs) promoted the expression of cyclooxygenase-2 (COX-2) and matrix metalloproteinase-9 (MMP-9), which further induced abnormal ESC growth, and initiated the invasion and implantation of the shed endometrium to the peritoneum $(7,8)$.

Macrophages have a key role in regulating and executing the immune response under various conditions. They are considered to be involved in highly complex immunological gynecological processes, including endometriosis, preeclampsia and miscarriage $(9,10)$. The activation and differentiation of macrophages is altered in these types of immune reactions. Higher levels of IDO1 in ectopic ESCs modulate adjacent macrophages through soluble factors, including interleukin (IL)-33, to generate a supportive microenvironment in endometriosis. Dysfunctional macrophages display reduced expression of Human Leukocyte Antigen-antigen D Related (HLA-DR) and CD11c, and increased secretion of modified cytokine IL-10 and transforming growth factor- $\beta 1$ (TGF- $\beta 1)$ (11). Thus, the cross-talk between ESCs and macrophages within the peritoneal cavity remains unclear. The present study aimed to investigate the effect of IDO1-induced tolerant macrophages on the survival of ESCs involved in the pathogenesis of endometriosis.

\section{Materials and methods}

Sample collection and cell culture. Patients (age, 23-40 years) that underwent laparoscopy and additional curettage for 
treatment of endometriosis $(n=16)$ or ovary dermoid cyst $(n=14)$ were originally enrolled in this study. Patients that were later considered to be negative for endometriosis $(n=4)$ or ovary dermoid cyst $(n=2)$ following laparoscopy and histological diagnosis were excluded from the study. In total, 12 endometrial and 12 endometriotic samples were obtained from patients who underwent laparoscopy for treatment of endometriosis or ovary dermoid cyst. Inclusion criteria were as follows: Reproductive age (23-40 years old); in the secretory phase of the menstrual cycle; absence of systemic pathologies; and no drug therapy in the past 6 months. Diagnosis was confirmed visually by laparoscopy and histological analysis. All of the women with endometriosis were classified as stage III/IV, according to the revised America Fertility Society classification of endometriosis (12). Peripheral blood samples $(15 \mathrm{ml})$ were collected sterilely in women with dermoid cyst $(n=12$; mean \pm SD: $33.6 \pm 6.2$ years old) as controls prior to the administration of general anesthesia with tracheal intubation in heparinized Hank's buffer solution (Gibco; Thermo Fisher Scientific, Inc.; Waltham, MA, USA). Endometriotic cyst wall tissue (ectopic endometrium) was obtained from ovarian endometriosis patients $(n=12$; mean \pm SD: $30.2 \pm 8.1$ years old $)$ during surgery, and normal endometrial samples were collected from control groups. The protocol was approved by the Research Ethics Committee of Nanjing Drum Tower Hospital (Nanjing, China) and informed written consent was obtained from all participants. All tissue samples, which were $\geq 200 \mathrm{mg}$, were collected under sterile conditions and transported to the laboratory on ice in Dulbecco's modified Eagle's medium (DMEM)/F-12 (Gibco; Thermo Fisher Scientific, Inc.). ESCs were purified as described previously (13). Immunocytochemistry identified $>95 \%$ vimentin-positive and cytokeratin-negative ESCs (13).

IDO1 overexpression or short hairpin RNA (shRNA) plasmid transfection. Normal ESCs were cultured in DMEM/F-12 with $10 \%$ fetal bovine serum in a 6 -well plate (FBS; Gibco; Thermo Fisher Scientific, Inc.). When cells had reached confluency, $10 \mu$ l Lipofectamine ${ }^{\circledR} 2000$ (Invitrogen; Thermo Fisher Scientific, Inc.), 2 ml OPTI-MEM ${ }^{\mathrm{TM}}$ (Gibco, Thermo Fisher Scientific, Inc.) and $4 \mu \mathrm{g}$ plasmid pEGFP-N1-IDO1 (Gene Chem Co., Ltd., Shanghai, China) or $4 \mu \mathrm{g}$ SD11-IDO1 shRNA (Gene Chem Co., Ltd.) were mixed and incubated at room temperature for $20 \mathrm{~min}$, and then added to the cells at room temperature according to manufacturer's protocol. The vector-only plasmid pEGFP-N1 and SD11 (Gene Chem Co., Ltd.) were used as negative controls, respectively. After $6 \mathrm{~h}$ incubation, cells were cultured in DMEM/F-12 containing $10 \% \mathrm{FBS}$ in $5 \% \mathrm{CO}_{2}$ at $37^{\circ} \mathrm{C}$.

Generation of human macrophages. Peripheral blood mononuclear cells (PBMC) were isolated from blood samples by Ficoll-Hypaque density gradient centrifugation (11). CD14 ${ }^{+}$ cells were obtained through positive selection by $\mathrm{CD} 14^{+}$ micromagnetic beads according to the manufacturer's instructions (Miltenyi Biotec GmbH, Bergisch Gladbach, Germany). Cell purity was identified as $>95 \%$ by flow cytometry identification using a fluorescein isothiocyanate (FITC)-anti CD14 monoclonal antibody (catalogue no. 555397; BD Biosciences, Franklin Lakes, NJ, USA). CD14 ${ }^{+}$cells were harvested at a final concentration of $2 \times 10^{7}$ cells $/ \mathrm{ml}$ and washed in cold
PBS. Then, $5 \mu \mathrm{l}$ CD14 monoclonal antibody was added into each $100 \mu \mathrm{l}$ of cell suspension for $15 \mathrm{~min}$ in the dark at room temperature. Following staining, cells were washed twice with cold PBS and then analyzed by Facs Calibur BD flow cytometry (BD Biosciences). Data were acquired in the list mode, and the relative proportions of cells within different areas of the fluorescence profile were quantified using the FlowJo 7.6 software program (FlowJo, LLC., Ashland, OR, USA). Monocytes were subsequently cultured with granulocyte macrophage colony-stimulating factor (GM-CSF; $5 \mathrm{ng} / \mathrm{ml}$; catalog no. 300-03-100, PeproTech, Rocky Hill, NJ, USA) and macrophage colony-stimulating factor (M-CSF; $20 \mathrm{ng} / \mathrm{ml}$; R\&D Systems, Inc., Minneapolis, MN, USA) in RPMI-1640 medium (Gibco; Thermo Fisher Scientific, Inc.) containing $10 \% \mathrm{FBS}$ and $2 \mathrm{mM} \mathrm{L-glutamine}$ for up to 6 days. The medium containing GM-CSF and M-CSF was changed every 3 days.

Cell co-culture unit. Normal, ectopic and transfected normal ESCs (ESCs transfected with pEGFP-N1-IDO1 plasmid or SD11-IDO1 shRNA) were cultured in 24-well plates (Corning Incorporated, Corning, NY, USA) at a density of $2 \times 10^{5}$ cells/well. After ESCs had reached confluency, the monocyte-generated macrophages were subsequently added to the wells directly at the same density as ESCs. After 48 h, following gentle scattering, the macrophages were collected. Some were immediately analyzed by flow cytometry for the phagocytosis assay; others were further co-cultured directly with normal ESCs for another $36 \mathrm{~h}$, and the normal ESCs were subsequently analyzed in cell viability, cell proliferation and Annexin V/propidium iodide (PI) apoptosis assays.

Phagocytosis assay. A total of $2 \times 10^{6}$ ESC-pretreated macrophages were mixed with fluoresbrite carboxy NYO-labeled beads in a ratio of 10:1 (1 $\mu$ m-diameter microspheres; Polysciences Inc., Warrington, PA, USA) for $30 \mathrm{~min}$ with shaking at $37^{\circ} \mathrm{C}$. The unbound beads were washed away by cold PBS (Corning Incorporation) twice and cells were then resuspended in 2\% bovine serum albumin (Sigma-Aldrich, Merck KGaA, Darmstadt, Germany). Then, the cell suspension was added to the upper layer of Ficoll-hypaque solution (GE Healthcare, Sunnyvale, CA, USA) at a ratio of 1:1, and centrifuged at $300 \times \mathrm{g}$ at room temperature for $10 \mathrm{~min}$. Using density-gradient centrifugation, the macrophages were separated in the Ficoll-hypaque solution layer. Following the resuspension of macrophages in PBS, the ratio of macrophages that ingested fluorescent beads was directly determined using Facs Calibur BD flow cytometry (BD Biosciences) and analyzed using the FlowJo 7.6 software program (FlowJo, LLC., Ashland, OR, USA).

Cell viability assay. To detect cell viability, an MTT (Sigma-Aldrich, Merck KGaA) assay was used. Normal ESCs ( $2 \times 10^{4}$ cell/per well in 96-well plate) were co-cultured with ESC-pretreated macrophages for $36 \mathrm{~h}$. Normal ESCs were subsequently incubated with $2.5 \mathrm{mg} / \mathrm{ml} \mathrm{MTT}$ for $4 \mathrm{~h}$, and $100 \mu \mathrm{l}$ dimethyl sulfoxide (Sigma-Aldrich; Merck, KGaA) was added. Absorbance (450 nm) was determined using the DigiScan Microplate Reader (ASYS Hitech GmbH, Eugendorf, Austria). These values were normalized to the measurement in normal ESCs co-cultured with macrophages that had not been 
previously cultures with ESCs, in which the absorbance was set at 1 .

Cell proliferation assay. Normal ESCs co-cultured with pretreated-macrophages were separated and detection by BrdU (5-bromo-2-deoxyuridine) Cell Proliferation Kit (Merck Millipore, Billerica, MA, USA) was performed for cell proliferation according to the manufacturer's instructions. The absorbance values (at $450 \mathrm{~nm}$ ) were detected by the DigiScan Microplate Reader (ASYS Hitech GmbH) and represent the rate of DNA synthesis, which corresponds to the number of proliferating cells. The values were normalized to the absorbance of normal ESCs co-cultured with untreated macrophages, which was set at 1 .

Measurement of apoptosis. The rate of apoptosis of co-cultured normal ESCs was analyzed by flow cytometry with Cell Apoptosis kit with Annexin V-FITC and propidium iodide for flow cytometry according to the manufacturer's protocol (Invitrogen, Thermo Fisher Scientific, Inc.). The relative proportions of cells within different areas of the fluorescence profile were quantified using the FlowJo 7.6 software program (FlowJo, LLC.).

Statistical analysis. One-way analysis of variance with the S-N-K method post hoc test was used for multiple comparisons, using SPSS software version 19.0 (IBM SPSS, Armonk, $\mathrm{NY}$, USA). $\mathrm{P}<0.05$ was considered to indicate a statistically significant difference.

\section{Results}

IDO1 in ectopic ESCs modulates macrophage phagocytosis. Our previous study demonstrated that IDO1 expression was higher in ectopic endometrial tissues compared with normal ones (7). To investigate the effect of IDO1 in ESCs on PBMC-derived macrophages, macrophages were pretreated with normal ESCs, ectopic ESCs, normal ESCs transfected with plasmid pEGFP-N1-IDO1, SD11-IDO1 shRNA, or vector-only plasmids. The aim of the current study was to determine whether IDO1 expression in ESCs is responsible for the phagocytic ability of macrophages. Compared with normal ESCs that had not been transfected (blank controls), pEGFP-N1 and SD11 vector-transfected ESCs (negative controls) had the same effect on macrophage phagocytosis ( $>0.05$; data not shown). IDO1 overexpression in normal ESCs significantly reduced the phagocytosis of co-cultured macrophages, compared with normal ESCs $(\mathrm{P}<0.05$; group c vs. group b; Fig. 1), whereas IDO1 interference demonstrated the opposite effects $(\mathrm{P}<0.01$; group d vs. group b; Fig. 1). Ectopic ESCs significantly inhibited the phagocytic capacity of co-cultured macrophages, compared with normal ESCs $(\mathrm{P}<0.01$; group e vs. group b; Fig. 1).

Ectopic ESC-pretreated macrophages increase the survival of ESCs via higher IDO1 expression in ESCs. To determine the effect of ESC-treated macrophages on viability and proliferation of ESCs, ESC-pretreated macrophages were separated and co-cultured with normal ESCs. pEGFP-N1 and SD11 vector-transfected ESC-educated macrophage (negative controls) had the same effect on ESC survival as normal ESCs (blank controls; data not shown). The viability and proliferation index of ESCs co-cultured with ectopic ESC-educated macrophages were 1.9- and 2.2-fold of the control, respectively $(\mathrm{P}<0.01$; ectopic ESC vs. normal ESC; Fig. 2). Significant increases in the viability and proliferation index of ESCs were observed when co-cultured with pEGFP-N1-IDO1-transfected ESC-induced macrophages compared with normal ones $(\mathrm{P}<0.05$; IDO1 overexpression vs. normal ESC; Fig. 2). However, the viability and proliferation index of ESCs co-cultured with pEGFP-N1-IDO1-transfected ESC-induced macrophage were significantly lower compared with those cultured with ectopic ESC-induced macrophages $(\mathrm{P}=0.017$, IDO1 overexpression vs. ectopic ESC in Fig. 2A; $\mathrm{P}=0.021$, IDO1 overexpression vs. ectopic ESC in Fig. 2B which indicated that ectopic ESC-educated macrophages may promote the growth of ESCs through a mediator other than IDO1.

Effect of IDO1-induced tolerant macrophages on the apoptosis of ESCs. ESCs co-cultured with ectopic ESC or pEGFP-N1-IDO1 transfected ESC-pretreated macrophages exhibited a significantly lower apoptosis rate compared with ESCs co-cultured with normal ESC-pretreated macrophages $(\mathrm{P}<0.01$; group e vs. group b and group c vs. group b; Fig. 3), and the apoptosis of ESCs increased when co-cultured with macrophages that were pretreated with SD11-IDO1 shRNA-transfected ESCs ( $\mathrm{P}<0.01$; group d vs. group b; Fig. 3).

\section{Discussion}

Endometriosis-associated inflammation is chronic and long lasting (14). An increasing number of studies have focused on the importance of immunological imbalances in women with endometriosis. It has been confirmed that, a permissive peritoneal environment may be associated with the initiation and development of endometriosis (1-3,11). Rather than effectively removing the retrograde endometrial fragments in the pelvic cavity, the tolerant environment facilitates the implantation, neo-angiogenesis and proliferation of ectopic endometrial tissue $(15,16)$. Conditions in the tolerant environment may include elevated levels of activated peritoneal macrophages, reduced natural killer cell activity, an abnormal T lymphocyte response and an increased number of regulatory $\mathrm{T}$ cells in endometriotic tissue and peritoneal fluid (17-20).

Cells of the monocyte-macrophage lineage are characterized by diversity and plasticity, which respond to environmental stimuli by acquiring diverse phenotypes. In response to external cues, M1 macrophage activation occurs with microbicidal and tumoricidal features. Alternatively, the M2 pathway predominantly participates in parasite containment, tissue remodeling and immunomodulation (21). The activated peritoneal macrophages have an important role in the onset and development of endometriosis (22). Impaired macrophages cannot effectively clear the ectopic endometrial cells in endometriosis patients (23), and inversely secrete various inflammatory mediators that may contribute to the progression of endometriosis. However, the understanding of the contribution of macrophages to the endometriotic environment remains inadequate. 
Phagocytic ability of macrophage

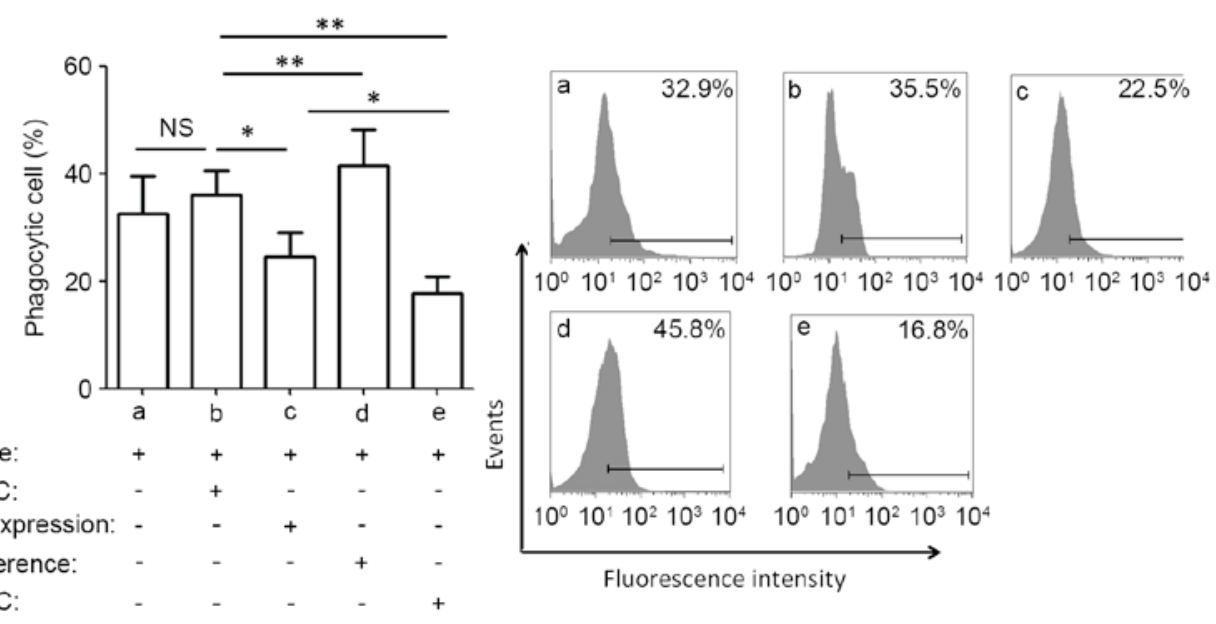

Figure 1. Phagocytic ability of macrophages in various ESC-macrophage co-culture groups. Normal ESCs, ectopic ESCs and normal ESCs treated with pEGFP-N1-IDO1 or SD11-IDO1 short hairpin RNA were co-cultured with human macrophages directly. The phagocytosis of macrophages was detected by flow cytometry. Phagocytic ability was significantly lower when macrophages were cultured with ectopic ESCs compared with normal ESCs. IDO1 overexpression in normal ESCs significantly decreased the phagocytosis of macrophages in the normal ESC-macrophage co-culture group, while IDO1 interference significantly increased it, compared with normal ESC-treated macrophages. Results are presented as the ratio of macrophages that ingested fluorescent beads, as the mean + standard deviation of 12 different experiments ( $\mathrm{P}<0.05$ and ${ }^{* *} \mathrm{P}<0.01$ ). ESC, endometrial stromal cells; IDO1, indoleamine 2,3-dioxygenase-1; NS, not significant; normal ESC, ESCs from patients without endometriosis; IDO1 overexpression, normal ESCs transfected with pEGFP-N1-IDO1; IDO1 interference, normal ESCs transfected with SD11-IDO1 short hairpin RNA; ectopic ESC, ESC from endometriosis-derived endometriotic tissue.

A

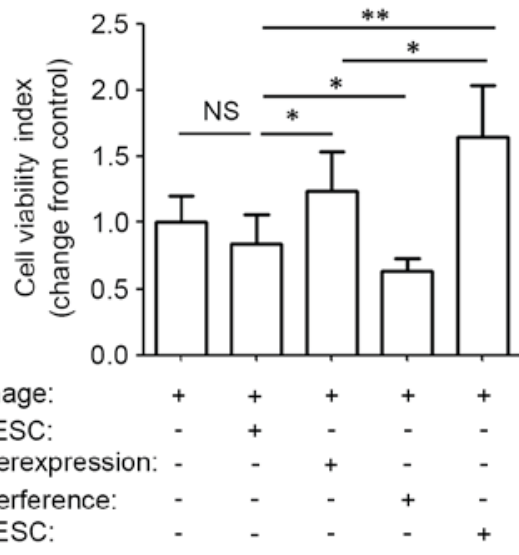

B

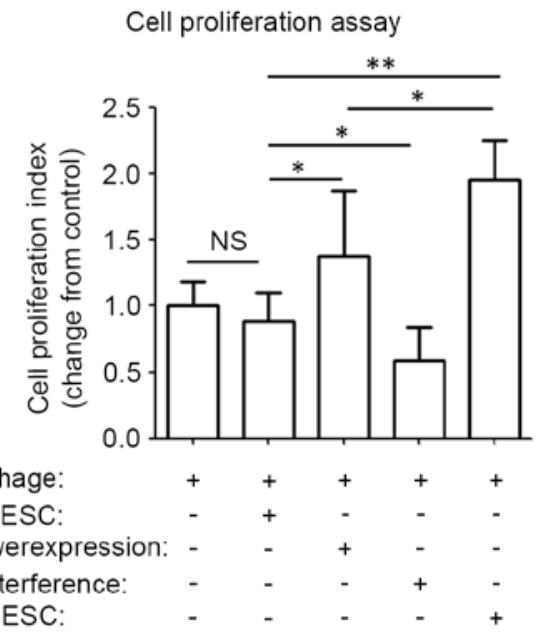

Figure 2. ESC-pretreated macrophages increase the survival of ESCs by IDO1. Peripheral blood mononuclear cell-derived macrophages were pretreated with normal ESCs, ectopic ESCs, IDO1-overexpressing and IDO1-deficient ESCs for $48 \mathrm{~h}$, and subsequently co-cultured with normal ESCs (2x104 cells/well in a 96-well plate) for $36 \mathrm{~h}$. (A) MTT assay and (B) 5-bromo-2-deoxyuridine ELISA assay were subsequently performed to analyze the viability and proliferation of normal ESCs, respectively. Results are presented as the mean \pm standard deviation of 12 different experiments $\left({ }^{*} \mathrm{P}<0.05\right.$ and $\left.{ }^{* *} \mathrm{P}<0.01\right)$. ESC, endometrial stromal cells; IDO1, indoleamine 2,3-dioxygenase-1; NS, not significant; normal ESC, ESCs from patients without endometriosis; IDO1 overexpression, normal ESCs transfected with pEGFP-N1-IDO1; IDO1 interference, normal ESCs transfected with SD11-IDO1 short hairpin RNA; ectopic ESC: ESCs from endometriosis-derived endometriotic tissue.

As it was previously demonstrated that the phenotype of PBMCs did not differ in women with and without endometriosis (24), PBMCs were obtained from control women in the present study. The current study co-cultured ESCs with macrophages to determine if IDO1 in ESCs had any effect on the immune function of macrophages. Following incubation with IDO1-overexpressing ESCs, macrophages exhibited a decreased phagocytic ability. Wu et al (25) and Chuang et al (26) demonstrated that the master regulator of the peritoneal microenvironment, prostaglandin E2 (PGE2), suppressed at least two aspects of the scavenger function of macrophages, including decreased secretion and activation of MMP-9, and reduced class B scavenger receptor (CD36) expression in peritoneal macrophages. Furthermore, PGE2 also inhibited annexin A2 expression, which led to a reduced immunological response by peritoneal macrophages (23). The present study subsequently investigated how the tolerant macrophages affected the growth of ESCs. The results demonstrated that the viability and proliferation of ESCs were significantly increased, and apoptosis index decreased, when co-cultured with macrophages pretreated with IDO1-overexpressing ESCs compared with macrophages treated with normal 

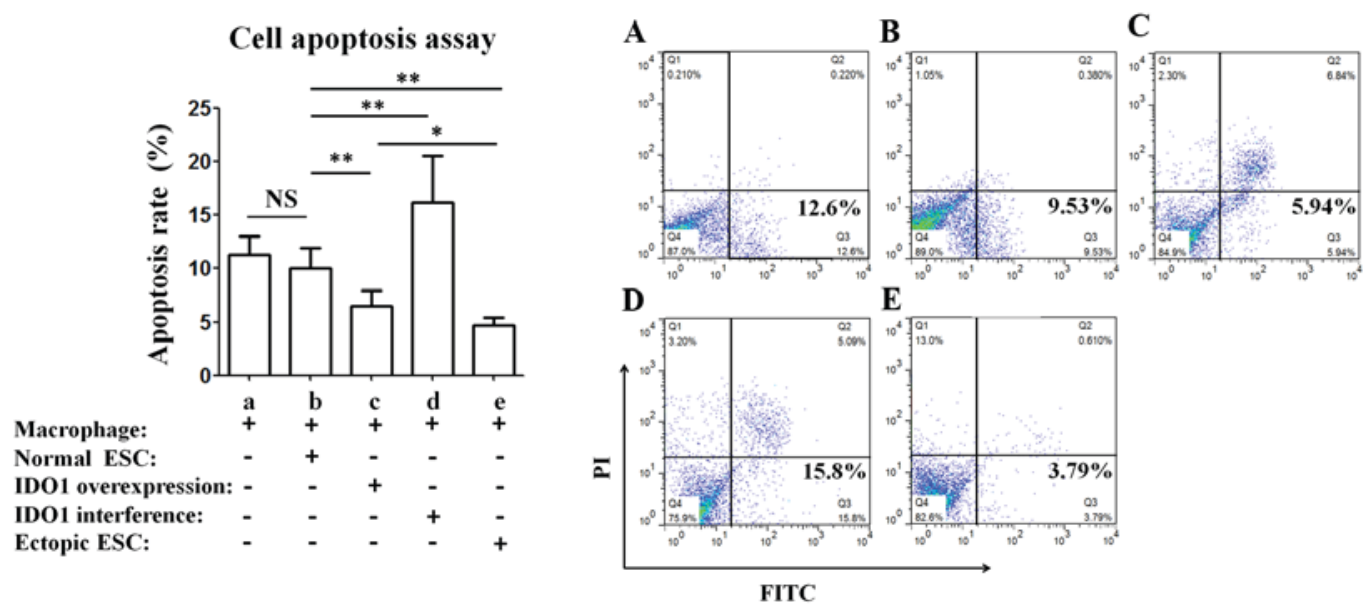

Figure 3. IDO1-induced tolerant macrophages inhibit the apoptosis of ESCs. Normal ESCs were co-cultured with peripheral blood mononuclear cell-derived macrophages, which were pretreated with normal ESCs, ectopic ESCs, normal ESCs transfected with plasmid pEGFP-N1-IDO1 or SD11-IDO1 short hairpin RNA. Subsequently, ESCs were collected and apoptosis was evaluated by flow cytometry. Results are presented as the mean + standard deviation of 12 different experiments $\left({ }^{*} \mathrm{P}<0.05,{ }^{* *} \mathrm{P}<0.01\right)$. ESC, endometrial stromal cells; IDO1, indoleamine 2,3-dioxygenase-1; NS, not significant; PI, propidium iodide; normal ESC, ESCs from patients without endometriosis; IDO1 overexpression, normal ESCs transfected with pEGFP-N1-IDO1; IDO1 interference, normal ESCs transfected with SD11-IDO1 short hairpin RNA; ectopic ESC, ESCs from endometriosis-derived endometriotic tissue.

ESCs. The results indicate that high levels of IDO1 expressed in the ectopic environment may induce the formation of tolerant macrophages, which in turn may promote ectopic ESC growth in the progression of endometriosis. There are various solute cytokines in the peritoneal microenvironment that are secreted by macrophages and may affect the survival of endometrial tissue and the development of endometriosis. Shi et al $(27,28)$ demonstrated that estradiol and 2,3,7,8-tetrachlorodibenzo-p-dioxin coordinated to the excessive growth of endometriotic cells in vitro by stimulating the secretion of pro-inflammatory cytokines, IL-8 and chemokine (C-C motif) receptor 8 (CCR8), by macrophages, which led to persistent and severe inflammation. Furthermore, increased RANTES in eutopic and ectopic ESCs recruited more macrophages into the environment, and also induced tolerance in macrophages, which inhibited the apoptosis and promoted the proliferation of ESCs in the endometriotic environment (3). Additionally, Khan et al (29) demonstrated that an inflammatory reaction in the intrauterine environment stimulated the expression of the stress reaction marker, human heat shock protein 70 (HSP70), which further stimulated the production of IL-6 and tumor necrosis factor $\alpha$ by macrophages, and promoted the proliferation of ESCs. However, these effects of HSP70 were more prominent in cells derived from women with endometriosis compared with normal ones. It may be inferred that macrophages from control women were less responsive to the inflammatory surrounding. This may be due to variations in cytokine secretion and the receptor-ligand binding affinity in macrophages of control women. The evidence discussed indicates that ESC-educated macrophages may regulate the growth of ESCs by secreting soluble cytokines. Further studies investigating the mechanism of the cross-talk between endometrial tissues and macrophages in the pelvis of women with endometriosis are required to strengthen the results of the present study.

In conclusion, the progression of endometriosis may be recognized as the product of evolving cross-talk between ectopic ESCs and macrophages within the peritoneal cavity. Increased IDO1 protein expression in eutopic and ectopic endometria of women with endometriosis is of biological importance. It may directly promote the proliferation and invasion of endometrial tissue by regulating the expression of COX-2 and MMPs. Additionally, it may also modulate adjacent macrophages to generate a supportive microenvironment. Dysfunctional macrophages exhibit impaired phagocytic abilities, which may lead to decreased clearance of endometriotic tissue outside the uterus. Furthermore, the altered polarization of macrophages may promote endometrial tissue growth and suppress its apoptosis by releasing a modified profile of cytokines, including IL-10 and TGF- $\beta 1$. Due to the potential role of IDO1 in endometriosis and its application in clinical trials of cancer therapy (30), further investigation is required to identify potential IDO1-based therapies for endometriosis.

\section{Acknowledgements}

The present study was supported by the National Natural Science Foundation of China (grant no. 81601354), the National Science Foundation of Jiangsu Province, China (grant no. BK20160128), and the Fundamental Research Funds for the Central Universities (grant no. 021414380180) (all to J. M.).

\section{References}

1. Burney RO and Giudice LC: Pathogenesis and pathophysiology of endometriosis. Fertil Steril 98: 511-519, 2012.

2. Pirdel L and Pirdel M: Role of iron overload-induced macrophage apoptosis in the pathogenesis of peritoneal endometriosis. Reproduction 147: R199-R207, 2014.

3. Wang XQ, Yu J, Luo XZ, Shi YL, Wang Y, Wang L and Li DJ: The high level of RANTES in the ectopic milieu recruits macrophages and induces their tolerance in progression of endometriosis. J Mol Endocrinol 45: 291-299, 2010.

4. Ravishankar B, Liu H, Shinde R, Chaudhary K, Xiao W, Bradley J, Koritzinsky M, Madaio MP and McGaha TL: The amino acid sensor GCN2 inhibits inflammatory responses to apoptotic cells promoting tolerance and suppressing systemic autoimmunity. Proc Natl Acad Sci USA 112: 10774-10779, 2015. 
5. Noh KT, Son KH, Jung ID, Kang TH, Choi CH and Park YM Glycogen synthase kinase-3 $\beta$ (GSK-3 $\beta$ ) inhibition enhances dendritic cell-based cancer vaccine potency via suppression of interferon- $\gamma$-induced Indoleamine 2,3-dioxygenase expression. J Biol Chem 290: 12394-12402, 2015.

6. Sucher R, Fischler K, Oberhuber R, Kronberger I, Margreiter C, Ollinger R, Schneeberger S, Fuchs D, Werner ER, Watschinger $\mathrm{K}$, et al: IDO and regulatory $\mathrm{T}$ cell support are critical for cytotoxic T lymphocyte-associated Ag-4 Ig-mediated long-term solid organ allograft survival. J Immunol 188: 37-46, 2012.

7. Mei J, Jin LP, Ding D, Li MQ, Li DJ and Zhu XY: Inhibition of IDO1 suppresses cyclooxygenase-2 and matrix metalloproteinase- 9 expression and decreases proliferation, adhesion and invasion of endometrial stromal cells. Mol Hum Reprod 18: 467-476, 2012

8. Mei J, Li MQ, Ding D, Li DJ, Jin LP, Hu WG and Zhu XY: Indoleamine 2,3-dioxygenase-1 (IDO1) enhances survival and invasiveness of endometrial stromal cells via the activation of JNK signaling pathway. Int J Clin Exp Pathol 6: 431-444, 2013.

9. Tang MX, Hu XH, Liu ZZ, Kwak-Kim J and Liao AH: What are the roles of macrophages and monocytes in human pregnancy? J Reprod Immunol 112: 73-80, 2015.

10. Lee CL, Guo Y, So KH, Vijayan M, Guo Y, Wong VH, Yao Y, Lee KF, Chiu PC and Yeung WS: Soluble human leukocyte antigen G5 polarizes differentiation of macrophages toward a decidual macrophage-like phenotype. Hum Reprod 30: 2263-2274, 2015

11. Mei J, Xie XX, Li MQ, Wei CY, Jin LP, Li DJ and Zhu XY: Indoleamine 2,3-dioxygenase-1 (IDO1) in human endometrial stromal cells induces macrophage tolerance through interleukin-33 in the progress of endometriosis. Int J Clin Exp Pathol 7: 2743-2757, 2014.

12. Revised American Fertility Society classification of endometriosis: 1985. Fertil Steril 43: 351-352, 1985.

13. Li MQ, Luo XZ, Meng YH, Mei J, Zhu XY, Jin LP and Li DJ: CXCL8 enhances proliferation and growth and reduces apoptosis in endometrial stromal cells in an autocrine manner via a CXCR1-triggered PTEN/AKT signal pathway. Hum Reprod 27: 2107-2116, 2012.

14. González-Ramos R, Defrère $S$ and Devoto L: Nuclear factor-kappaB: A main regulator of inflammation and cell survival in endometriosis pathophysiology. Fertil Steril 98 520-528, 2012

15. Matarese G, De Placido G, Nikas Y and Alviggi C: Pathogenesis of endometriosis: Natural immunity dysfunction or auto-immune disease? Trends Mol Med 9: 223-228, 2003.

16. Barrier BF: Immunology of endometriosis. Clin Obstet Gynecol 53: 397-402, 2010

17. Budiu RA, Diaconu I, Chrissluis R, Dricu A, Edwards RP and Vlad AM: A conditional mouse model for human MUC1-positive endometriosis shows the presence of anti-MUC1 antibodies and Foxp3+regulatory T cells. Dis Model Mech 2: 593-603, 2009.
18. Berbic M, Hey-Cunningham AJ, Ng C, Tokushige $\mathrm{N}$, Ganewatta S, Markham R, Russell P and Fraser IS: The role of Foxp3+ regulatory T-cells in endometriosis: A potential controlling mechanism for a complex, chronic immunological condition. Hum Reprod 25: 900-907, 2010.

19. Olkowska-Truchanowicz J, Bocian K, Maksym RB, Białoszewska A, Włodarczyk D, Baranowski W, Ząbek J, Korczak-Kowalska G and Malejczyk J: CD4+ CD25+ FOXP3+ regulatory $\mathrm{T}$ cells in peripheral blood and peritoneal fluid of patients with endometriosis. Hum Reprod 28: 119-124, 2013.

20. Li MQ, Wang Y, Chang KK, Meng YH, Liu LB, Mei J, Wang Y, Wang XQ, Jin LP and Li DJ: CD4+Foxp3+ regulatory T cell differentiation mediated by endometrial stromal cell-derived TECK promotes the growth and invasion of endometriotic lesion. Cell Death Dis 5: e1436, 2014.

21. Sica A and Mantovani A: Macrophage plasticity and polarization: In vivo veritas. J Clin Invest 122: 787-795, 2012.

22. Khoufache K, Bazin S, Girard K, Guillemette J, Roy MC, Verreault JP, Al-Abed Y, Foster W and Akoum A: Macrophage migration inhibitory factor antagonist blocks the development of endometriosis in vivo. PLoS One 7: e37264, 2012

23. Wu MH, Chuang PC, Lin YJ and Tsai SJ: Suppression of annexin $\mathrm{A} 2$ by prostaglandin $\mathrm{E}_{2}$ impairs phagocytic ability of peritoneal macrophages in women with endometriosis. Hum Reprod 28: 1045-1053, 2013.

24. Yamamoto Y, Maeda N, Izumiya C, Kusume T, Oguri H, Kawashima M, Hayashi K, Nomura A, Yamashita C and Fukaya T: Decreased human leukocyte antigen-DR expression in the lipid raft by peritoneal macrophages from women with endometriosis. Fertil Steril 89: 52-59, 2008

25. Wu MH, Shoji Y, Wu MC, Chuang PC, Lin CC, Huang MF and Tsai SJ: Suppression of matrix metalloproteinase-9 by prostaglandin $\mathrm{E}(2)$ in peritoneal macrophage is associated with severity of endometriosis. Am J Pathol 167: 1061-1069, 2005.

26. Chuang PC, Lin YJ, Wu MH, Wing LY, Shoji Y and Tsai SJ: Inhibition of CD36-dependent phagocytosis by prostaglandin E2 contributes to the development of endometriosis. Am J Pathol 176: 850-860, 2010.

27. Shi YL, Luo XZ, Zhu XY, Hua KQ, Zhu Y and Li DJ: Effects of combined 17beta-estradiol with TCDD on secretion of chemokine IL-8 and expression of its receptor CXCR1 in endometriotic focus associated cells in co-culture. Hum Reprod 21: 870-879, 2006.

28. Shi YL, Luo XZ, Zhu XY and Li DJ: Combination of 17beta-estradiol with the environmental pollutant TCDD is involved in pathogenesis of endometriosis via up-regulating the chemokine I-309-CCR8. Fertil Steril 88: 317-325, 2007.

29. Khan KN, Kitajima M, Inoue T, Tateishi S, Fujishita A, Nakashima $\mathrm{M}$ and Masuzaki H: Additive effects of inflammation and stress reaction on Toll-like receptor 4-mediated growth of endometriotic stromal cells. Hum Reprod 28: 2794-2803, 2013.

30. Soliman H, Mediavilla-Varela $\mathrm{M}$ and Antonia S: Indoleamine 2,3-dioxygenase: Is it an immune suppressor? Cancer J 16: 354-359, 2010. 